\begin{tabular}{|l|l|l|l|l|l|}
\hline Intropica & ISSN 1794-161X & Vol. 13 & No.1 & $51-56$ & Santa Marta-Colombia, enero-junio de 2018 \\
\hline
\end{tabular}

\title{
COMPUESTOS NITROGENADOS EN LOS SEDIMENTOS DEL ESTERO SAN CAMILO: GUAYAS, ECUADOR Y SU RELACIÓN CON ACTIVIDADES ANTRÓPICAS ADYACENTES
}

\author{
NITROGEN COMPOUNDS IN THE SEDIMENTS OF THE SAN CAMILO MAT: GUAYAS, \\ ECUADOR AND ITS RELATIONSHIP WITH ADJACENT ANTHROPIC ACTIVITIES
}

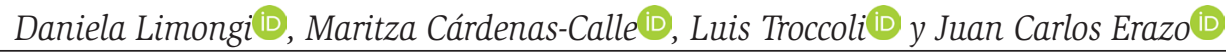

\begin{abstract}
RESUMEN
En la provincia del Guayas se encuentra la mayor población urbana del Ecuador con 3645483 habitantes y durante la última década el crecimiento urbano se ha concentrado en el cantón Durán. En ese sentido, las actividades antrópicas se han derivado en urbanismo, al desarrollo agrícola y el industrial, por lo que se han alterado las condiciones normales de los ecosistemas acuáticos adyacentes. En el presente estudio, se determinó la presencia de nitratos, nitritos y amonio en los sedimentos del Estero San Camilo (adyacente al cantón o pueblo de Durán) en noviembre de 2015 durante la época seca. Se realizaron muestreos de sedimentos en cuatro estaciones considerando la principal actividad productiva del área (industrial, urbana y agrícola), en las cuales se recolectaron tres réplicas mediante el uso de una draga Van Veen. Se registraron variaciones espaciales significativas $(\mathrm{p}<0,05)$ entre las concentraciones promedio de los compuestos nitrogenados (amonio 27,7 $\pm 2,3 \mathrm{mg} / \mathrm{kg} ;$ nitrito 0,64 \pm 0,06 $\mathrm{mg} / \mathrm{kg}$ y nitrato $54 \pm 2,7 \mathrm{mg} / \mathrm{kg}$ ). Los nitratos y nitritos registraron las mayores concentraciones promedios en la estaciones con influencia urbana. Mientras que el amonio presentó los valores más elevados en la estación con mayor influencia de zonas agrícolas. Lo que evidencia la actividad urbana como el mayor efecto contaminante.
\end{abstract}

PALABRAS CLAVE: estuarios; eutrofización; nitrógeno; sedimentos estuarinos; urbanismo

\begin{abstract}
Guayas province is the largest urban population of Ecuador with 3645483 inhabitants and during the last decade urban growth has been concentrated in the canton Duran In that sense, the anthropic activities have been derived in urbanism, agricultural development, and Industrial environment, which has altered the normal conditions of adjacent aquatic ecosystems. In the present study, the presence of nitrates, nitrites, and ammonium in the sediments of Estero San Camilo (adjacent to Durán) was determined in November 2015 during the dry season. Sediment sampling was carried out in four stations considering the main productive activity of the area (industrial, urban and agricultural), in which three replicates were collected using a Van Veen dredger. There were significant spatial variations $(\mathrm{p}<0.05)$ between the average concentrations of nitrogen compounds (ammonium $27.7 \pm 2.3 \mathrm{mg} / \mathrm{kg}$, nitrite $0.64 \pm 0.06 \mathrm{mg} / \mathrm{kg}$ and nitrate $54 \pm 2.7 \mathrm{mg} / \mathrm{kg}$ ). Nitrates and nitrites recorded the highest average concentrations in stations with the urban influence. While the ammonium presented the highest values in the season with greater influence of agricultural zones. This shows the urban activity as the major polluting effect.
\end{abstract}

KEY WORDS: estuaries; eutrophication; nitrogen; estuarine sediments; urbanism

Dirección de los autores:

Facultad de Artes Liberales y Ciencias de la Educación-Escuela de Ciencias Ambientales, Universidad Espíritu Santo-Ecuador, km. 2,5 Vía Puntilla Samborondón, Ecuador; e-mail: mdlimongi@uees.edu.ec (D.L.); macardenas@uees.edu.ec (M.C.C.); jucerazo@yahoo.com (J.C.E.). Universidad de Oriente. Instituto de Investigaciones Científicas. Isla de Margarita. Venezuela. e-mail: luis.troccoli@gmail.com (L.T.). 
La contaminación por el exceso de nitrógeno en ecosistemas acuáticos representa un serio problema ambiental en humedales, estuarios y cuencas hidrográficas, en donde se desarrollan diversas actividades antrópicas productivas como la agricultura, la ganadería y la extracción minera. Amonio $\mathrm{NH}_{4}$, nitrito $\mathrm{NO}_{2}$ y nitrato $\mathrm{NO}_{3}$ constituyen los compuestos nitrogenados más comunes en ecosistemas (Camargo y Alonso, 2007). Al respecto, se ha señalado la necesidad de evaluar los efectos de actividades humanas en ecosistemas acuáticos de Latinoamérica (Erisman et al., 2013) y en zonas costeras de Ecuador como señalan Twilley et al. (2001).

El Estero San Camilo, se encuentra ubicado al noroeste del cantón (pueblo) Durán (uno de los cantones con mayor crecimiento industrial) en la provincia del Guayas, en la costa ecuatoriana, y forma parte del sistema hidrográfico de la cuenca del Río Guayas (Páez, 2011; CAAM, 1996). Al igual que otros cuerpos hídricos, se encuentran afectados por vertidos sin previo tratamiento de las aguas industriales como consecuencia, acompañada de la emisión continua de residuos domésticos, ha originado la disminución de la diversidad de especies de invertebrados y peces, provocando cambios en la composición de las comunidades biológicas, desarrollo de especies invasoras, floraciones algales, reducción de la transparencia del agua y aumento de procesos anaeróbicos que desarrollan olores ofensivos (Blondel et al., 2010; Consesa y Lenzi, 2010; Benjumea-Hoyos et al., 2014; Cobeña, 2015; Cárdenas-Calle, 2016).

Los estudios en el estero y en general en Ecuador, han sido escasos y focalizados a evaluar la calidad de la columna de agua, quedando en un segundo plano la evaluación de los sedimentos estuarinos, los cuales sirven como sumidero de contaminantes. A pesar de su importancia aún se carece de una normativa ambiental para que las autoridades exijan su monitoreo permanente (Cárdenas-Calle, 2016). En ese sentido, no existe información sobre la presencia de compuestos nitrogenados en sedimentos del área. Sin embargo, existen pocos estudios que muestran alteraciones de sus aguas, como la detección de concentraciones de oxígeno disuelto $<5 \mathrm{mg}$ / L consideradas inferiores a las exigidos por la norma de calidad admisible (Cobeña, 2015). Además, se ha evidenciado en la zona, sensibilidad de macroinvertebrados al aplicar el Índice Biótico BMWP (Cárdenas-Calle, 2016). El objetivo de este estudio fue evaluar las tasas de concentraciones de $\mathrm{NO}_{3}$ y $\mathrm{NO}_{2}$ y $\mathrm{NH}_{4}$ presentes en los sedimentos del Estero San Camilo, a lo largo de áreas con influencia industrial, agrícola y asentamientos humanos. En el Estero San Camilo se ubicaron las siguientes coordenadas para cada zona de estudio. La zona industrial: Estación 1 y 3 (Estación 1, latitud: $2^{\circ} 13^{\prime} 14^{\prime \prime} S$ y longitud: $79^{\circ} 49^{\prime} 32^{\prime \prime}$ E; Estación 3, latitud: $2^{\circ} 12^{\prime} 40^{\prime \prime} \mathrm{S}$ y longitud: $79^{\circ} 48^{\prime} 55^{\prime \prime} \mathrm{E}$ ). La zona con asentamientos humanos: Estación 2 (latitud: $2^{\circ} 12^{\prime} 42^{\prime \prime} \mathrm{S}$ y longitud: $79^{\circ} 49^{\prime} 2$ ” E) y la zona donde se produce y almacena alimentos balanceados: Estación 4 (latitud: $2^{\circ} 12^{\prime} 42^{\prime \prime} \mathrm{S}$ y longitud: $79^{\circ} 48^{\prime} 34^{\prime \prime} \mathrm{E}$ ). El área estudiada abarcó una extensión aproximada de $3 \mathrm{~km}$ desde su inicio hasta la desembocadura del Río Guayas, a 4 km de distancia de la ciudad de Guayaquil.

Los sitios de muestreo se ubicaron aleatoriamente con una distancia en metros diferenciada entre cada uno al nivel del mar, en un área donde el promedio de precipitación anual es de $338,30 \mathrm{~mm}$ y la temperatura promedio anual es de $28,05^{\circ} \mathrm{C}$ y las principales actividades que se desarrollan en sus márgenes son: agrícola, urbanística e industrial (Páez, 2011; INAMHI, 2015).

Se recolectaron los sedimentos (tres réplicas) mediante el uso de la draga de Van Veen (capacidad de 0,1 $\mathrm{m}^{3}$ ), en cuatro estaciones durante la bajamar el 23 de noviembre de 2015 (temporada seca en Ecuador), obteniéndose 12 muestras. Estas almacenadas en bolsas plásticas herméticas y mantenidas en refrigeración hasta el ingreso al laboratorio CESTTA de la Escuela Superior Politécnica del Chimborazo, antes de las 12 horas pasada su recolección. La valoración de las concentraciones de nitrito $\mathrm{NO}_{2}$, nitrato $\mathrm{NO}_{3}$ y amonio $\mathrm{NH}_{4}$ fueron realizadas usando el método espectrofotométrico (APHA - WPCF, 1999).

Para evaluar la existencia de diferencias significativas entre las estaciones de estudio se realizó un análisis de varianza no paramétrico (Kruskal Wallis) luego de comprobar el no cumplimiento de los supuestos de normalidad y homocedasticidad, para determinar diferencias significativas entre estaciones, de las concentraciones de los compuestos nitrogenados para ello se usó el software estadístico Statgraphics Plus versión 5.1. Se realizó estadística multivariada, se efectuó un análisis de conglomerados para determinar la similitud de las estaciones con respecto a las concentraciones de compuestos nitrogenados en las distintas estaciones de muestreo. Este análisis se basó en el método de mínima varianza de Ward’s y la distancia métrica Euclidiana (Zar, 1996), Además, se realizó un análisis de componentes principales (ACP) para 
determinar la relación entre las variables en las zonas de muestreo (Johnson y Wichern, 1992) mediante el uso del software estadístico Infostat versión libre 2011.

Los componentes nitrogenados mostraron diferencias significativas entre las estaciones muestreadas. El promedio de concentración más alto de nitratos osciló entre 61,42 a $(54,41 \mathrm{mg} / \mathrm{kg})$ y nitritos $(0,87$ $\mathrm{mg} / \mathrm{kg}$ ) se detectó en la zona anexa a asentamientos urbanos (estación 2), mientras que promedio más alto de amonio (27,78 mg / kg) se detectó en zona de influencia agrícola (Tabla 1).

Tabla 1. Parámetros estadísticos (promedio, desviación estándar, mínimo y máximo) de nitratos, nitritos y amonio en sedimento (mg / kg) por estación de muestreo.

\begin{tabular}{|c|c|c|c|c|c|c|}
\hline \multirow{2}{*}{ Estaciones } & \multicolumn{2}{|c|}{ Nitrato (mg / kg) } & \multicolumn{2}{|c|}{ Nitrito (mg / kg) } & \multicolumn{2}{|c|}{ Amonio (mg / kg) } \\
\hline & $(\mathrm{X} \pm \mathrm{DE})$ & Min - Max & $(\mathrm{X} \pm \mathrm{DE})$ & Min - Max & $(\mathrm{X} \pm \mathrm{DE})$ & Min - Max \\
\hline 1 & $48,84 \pm 5,77$ & $53,31-42,33$ & $0,61 \pm 4,39$ & $0,68-0,57$ & $22,91 \pm 1,56$ & $24,29-21,21$ \\
\hline 2 & $66,30 \pm 4,39$ & $71,33-63,24$ & $0,87 \pm 0,05$ & $0,92-0,82$ & $26,55 \pm 5,62$ & $30,44-20,10$ \\
\hline 3 & $56,04 \pm 7,60$ & $61,42-47,34$ & $0,76 \pm 0,21$ & $0,93-0,53$ & $22,38 \pm 1,19$ & $23,74-21,50$ \\
\hline 4 & $46,48 \pm 6,86$ & $53,68-40,01$ & $0,32 \pm 0,13$ & $0,47-0,25$ & $39,27 \pm 6,33$ & $43,19-42,66$ \\
\hline
\end{tabular}

El análisis de conglomerados (Figura 1), permitió identificar tres grupos bien definidos, siendo los más similares las estaciones 2 (influenciada por asentamientos humanos) y 3 (influenciada por la actividad industrial) y la estación con mayor disimilitud fue la 4 (influenciada por actividades agrícolas).

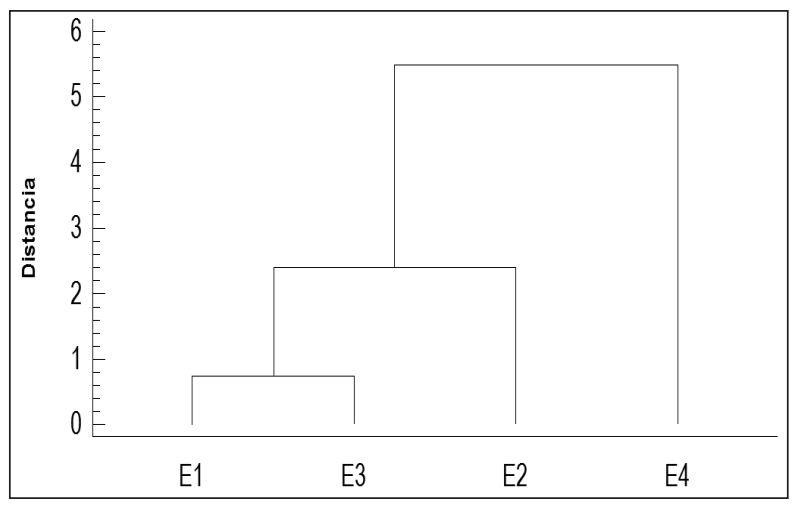

Figura 1. Dendograma de similaridad que muestra la asociación entre las muestras recolectadas.

El análisis de componentes principales, determinó que los tres parámetros analizados suman el $96,8 \%$ de la variabilidad explicada de los datos. El amonio es la variable con mayor correlación con el componente 1, en sentido inverso al nitrito y nitrato. El análisis de Kruskal Wallis, mostró diferencias significativas entre las zonas de actividad antrópica $(\mathrm{p}<0,05)$ a nivel de las concentraciones de nitratos, nitritos y amonio en los sedimentos de las estaciones muestreadas con (figura 2).

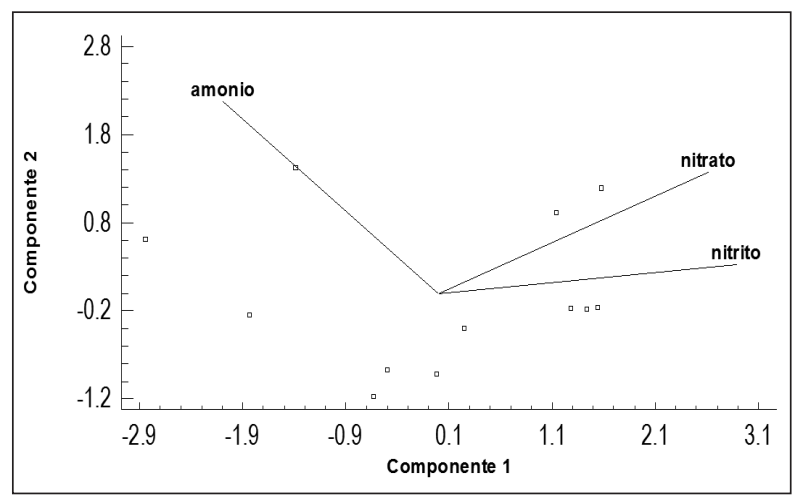

Figura 2. Proyección ortogonal de los dos primeros componentes del Análisis de Componentes Principales.

Los resultados obtenidos muestran que la variación espacial significativa de los compuestos nitrogenados en los sedimentos del Estero San Camilo, está relacionada al tipo de actividades productivas, en el caso de la estación con incidencia de asentamientos humanos (estación 2) y actividad industrial (estación 3) los valores de nitratos y nitritos fueron muy similares ya que la presión antrópica de ambos sectores provenía de efluentes de aguas domésticas e industriales descargadas directamente al estuario. Es importante destacar al respecto, que la presencia de 
concentraciones de nitrito ( $>0,5 \mathrm{mg} / \mathrm{kg}$ ) y amonio ( $>10 \mathrm{mg} / \mathrm{kg}$ ) son indicadores de niveles eutróficos (Islam et al., 2015).

Las actividades agrícolas e industriales (producción de balanceados) desarrolladas en la estación 1 y 4 respectivamente, presentaron concentraciones más bajas de nitratos que las áreas con influencia doméstica e industrial. Al respecto se sugiere que el tiempo de residencia permite la oxidación de los compuestos nitrogenados (Aranda, 2004; Guieysse y Norvill, 2014). En el caso de las aguas de las actividades agrícolas, probablemente se diluyen hasta llegar a la cuenca baja y en la zona de desembocadura del estero, existiendo influencia de fuentes difusas, por lo que es difícil cuantificar el aporte de nutrientes en los cuerpos de agua (Aranda, 2004).

En contradicción con lo mencionado, los estudios realizados por Aranda (2004), en México, muestran que las actividades agrícolas contribuyeron en un $34 \%$, con el nitrógeno aportado por las actividades antrópicas lo que constituye el mayor aporte en el proceso de eutrofización, creando desequilibrio en los procesos biogeoquímicos de los cuerpos de agua que exceden su capacidad de carga (Cobeña, 2015; Echaniz y Vignatti, 2009).

Respecto a la diferencia de concentraciones entre los diferentes parámetros muestreados, el compuesto nitrogenado más estable es el nitrato por efecto de la oxidación (Anton y Lizaso, 2003), siendo el compuesto de mayores registros. En su proceso biológico el amonio se oxida a nitrito y luego a nitrato, este proceso junto con la degradación de la materia orgánica, provoca disminución en las concentraciones de niveles de oxígeno disuelto producto del exceso de nutrientes en cuerpos de agua (Aranda, 2004; Guieysse y Norvill, 2014). Por ello, es importante el muestreo en sedimentos, considerando que funcionan como reservorio de nutrientes en estuarios y costas, y su tiempo de residencia es mayor que en el agua (Hayn et al., 2014; Estigoni et al., 2017). Además, los nutrientes concentrados en los sedimentos, se infiltran hasta llegar a los acuíferos (Lin y Dushoff, 2004).

Por otra parte, la eutrofización, en estuarios afecta la macrofauna. En este sentido, Nicastro y Bishop (2013) detectaron que las macrofauna en estuarios australianos varían de acuerdo al balance de nutrientes de los mismos. Como consecuencia detectaron disminución de macroinvertebrados acuáticos presentes en los sedimentos.

En estudio realizado en el Estero San Camilo y aplicando el índice biótico Biological Monitoring working Party (BMWP), se determinó que el cuerpo de agua estaba fuertemente contaminado con aguas residuales y su condición es muy crítica (Cárdenas-Calle, 2016). Entre los efectos resaltantes de elevadas concentraciones de nutrientes, se genera exceso de materia orgánica causando eutrofización, con niveles de anoxia en el fondo del estuario y producir la muerte de los organismos bentónicos (Rabalais et al., 2009).

La gran concentración de amonio, detectada en la estación 4, pudo generarse debido a que en esa zona se construye un nuevo parque industrial, y se usan explosivos, que contienen nitrato de amonio $\left(\mathrm{NH}_{4} \mathrm{NO}_{3}\right)$, y podría infiltrarse o lixiviarse mientras como consecuencia del proceso (Reimer et al., 2015). Además esta estación recibe los afluentes de zonas agrícolas que utilizan fertilizantes con contenido de nitrógeno como: nitrato de amonio $\left(\mathrm{NH}_{4} \mathrm{NO}_{3}\right)$, sulfato de amonio $\left(\left(\mathrm{NH}_{4}\right)_{2} \mathrm{SO}_{4}\right)$, urea $\left(\mathrm{CH}_{4} \mathrm{~N}_{2} \mathrm{O}\right)$ (Carpenter y Capone, 1983; IPNI, 2015); estos aportes agrícolas llegan a la desemboca en el Río Guayas (estación 1), donde hay mayor remoción de las aguas, por esto podría aumentar la dilución de los compuestos nitrogenados disminuyendo las concentraciones de los mismos (Echaniz y Vignatti, 2009).

Las presencia de amonio y nitritos en concentraciones elevadas en sedimentos del Estero San Camilo son indicadores de contaminación orgánica (Carpenter y Capone, 1983). Al respecto, existe variación espacial de los compuestos nitrogenados influenciadas por las diversas actividades productivas que se realizan en las riveras del área de estudio, sin control de los vertidos (Cobeña, 2015) lo que sugiere que se esté generando un deterioro de la calidad de este cuerpo hídrico.

Sería recomendable que ante la detección de concentraciones elevadas en los sedimentos del estuario, que el Ministerio del Ambiente de Ecuador, inicie la implementación de una normativa para evaluar los niveles máximos permitidos de nitratos, nitritos y amonio en sedimentos. De esa manera, se podrán controlar de forma efectiva, las descargas de aguas residuales industriales y domésticas para prevenir el impacto ambiental en comunidades bentónicas (Sany et al., 2014; Li et al., 2017). 


\section{REFERENCIAS}

Antón, A. y Lizaso, J. 2003. Nitritos, nitratos y nitrosaminas. En: Fundisa. Artículos de divulgación. Fundación Ibérica para la seguridad alimentaria, Madrid.

APHA - WPCF. 1999. Standard methods for the examination of water and wastewater. 20th edition. American Public Health Association, USA.

Aranda, N. 2004. Eutrofización y Calidad del agua de una zona costera tropical. Tesis de Doctorado, Department d'Ecologia, Universitat de Barcelona, Barcelona, España.

Benjumea-Hoyos, C.A., Bedoya-Castaño, C.L. y ÁlvarezAlzate, D.A. 2014. Evolución de la carga de nutrientes de ríos de montaña que fluyen a un embalse, Cuenca media del río Magdalena. Revista EIA 11: 77-91.

Blondel, J., Aronson, J. y Bodiou, J. 2010. Mediterranean Region: Biological Diversity Through Time and Space. 2nd Edition. Oxford, Inglaterra.

CAAM, B.J. 1996. Desarrollo y problemática ambiental del área de Golfo de Guayaquil. CREARIMAGEN, Ecuador.

Camargo, J.A. y Alonso, A. 2007. Contaminación por nitrógeno inorgánico en los ecosistemas acuáticos: problemas mediaoambientales, criterios de calidad del agua, e implicaciones del cambio climático. Ecosistemas 16(2): 98-110.

Cárdenas-Calle, M. 2016. The community structure of benthic macroinvertebrates in the inner branches of Estero Salado Estuary and Guayas River in Ecuador. Heriot watt University School of Life Sciences, Edinburgh.

Carpenter, E.J. y Capone, D.G. 1983. Nitrogen in the marine environment. Elsevier, New York.

Cobeña, M. 2015. Auditoría ambiental de cumplimiento construcción, montaje y operación de la maquinaria papelera 1 MP-1 de la compañía SURPAPELCORP S.A. Prefectura del Guayas, Ecuador.

Consesa, G.C. y Lenzi, M.A. 2010. Check Dams, Morphological Adjustments and Erosion Control in Torrential Streams. Hauppauge. Nova Science Publishers, USA.

Echaniz, S. y Vignatti, A. 2009. Determinación del estado trófico y de la capacidad de carga del embalse Casa de Piedra. BioScriba 2(1): 41-51.
Erisman, J.W., Galloway, J.N., Seitzinger, S., Bleeker, A., Dise, N.B., Petrescu, A.R., Leach, A.M. y de Vries, W. 2013. Consequences of human modification of the global nitrogen cycle. Philosophical Transactions of the Royal Society B 368(1621): 20130116.

Estigoni, M., Miranda, R. y Mauad, F. 2017. Hydropower reservoir sediment and water quality assessment. Management of Environmental Quality: An International Journal 28 (1): 43-56.

Guieysse, B. y Norvill, Z.N. 2014. Sequential chemicalbiological processes for the treatment of industrial wastewaters: review of recent progresses and critical assessment. Journal of hazardous materials 267: 142-152.

Hayn, M., Howarth, R., Marino, R., Ganju, N., Berg, P., Foreman, K. H., Giblin, A.E. y McGlathery, K. 2014. Exchange of nitrogen and phosphorus between a shallow lagoon and coastal waters. Estuaries and coasts 37(1): 63-73.

Instituto Nacional de Meteorología e Hidrología INAMHI. 2015. Anuario Meteorológico 2012. No 52. Instituto Nacional de Meteorología e Hidrología, Quito, Ecuador.

International Plant Nutrition Institute - IPNI. 2015. Fuentes de Nutrientes-Específicos. IPNI_URL:_https://www.ipni. net/specifics-es. Consultado: 05 de noviembre 2016.

Islam, M.S., Ahmed, M.K., Raknuzzaman, M., HabibullahAl-Mamun, M. e Islam, M.K. 2015. Heavy metal pollution in surface water and sediment: a preliminary assessment of an urban river in a developing country. Ecological Indicators 48: 282-291.

Johnson, R. y Wichern, D. 1992. Applied multivariate statistical analysis. 3rd edition. Prentice-Hall Int., New Jersey, USA.

Li, Y., Xu, E.G., Liu, W., Chen, Y., Liu, H., Li, D., Liu, Z., Giesy, J.P. y Yu, H. 2017. Spatial and temporal ecological risk assessment of unionized ammonia nitrogen in Tai Lake, China (2004-2015). Ecotoxicology and Environmental Safety 140: 249-255.

Lin, B.B. y Dushoff, J. 2004. Mangrove filtration of anthropogenic nutrients in the Rio Coco Solo, Panama. Management of Environmental Quality: An International Journal 15(2): 131-142.

Nicastro, A. y Bishop, M.J. 2013. Weak and habitatdependent effects of nutrient pollution on macrofaunal communities of southeast Australian Estuaries. PloS one 8(6): e65706. 
Páez, K. 2011. Plan de desarrollo territorial del canton Eloy Alfaro Durán. AME, Ecuador.

Rabalais, N., Turner, R., Díaz, R. y Justic, D. 2009. Global Change and eutrofication of coastal waters. ICES Journal of Marine Science 66: 1528-1537.

Reimer, J.D., Yang, S.Y., White, K.N., Asami, R., Fujita, K., Hongo, C., Ito, S., Kawamura, I., Maeda, I., Mizuyama, M., Obuchi, M., Sakamaki, T., Tachihara, K., Tamura, M., Tanahara, A. Yamaguchi, A. y Jenke-Kodama, H. 2015. Effects of causeway construction on environment and biota of subtropical tidal flats in Okinawa, Japan. Marine pollution bulletin 94 (1): 153-167.
Sany, S.B.T., Hashim, R., Rezayi, M., Salleh, A. y Safari, O. 2014. A review of strategies to monitor water and sediment quality for a sustainability assessment of marine environment. Environmental Science and Pollution Research 21(2): 813-833.

Twilley, R.R., Cárdenas, W., Rivera-Monroy, V.H., Espinoza, J., Suescum, R., Armijos, M.M. y Solórzano, L. 2001. The Gulf of Guayaquil and the Guayas river estuary, Ecuador. En: Seeliger, U. y Kjerfve, B., Editores. Coastal Marine Ecosystems of Latin America. Springer, Berlin, Heidelberg.

Zar, J. 1996. Biostatistical Analysis. Prentice Hall, New Jersey.

Fecha de recepción: 03/02/2018

Fecha de aceptación: 16/03/2018

Publicado en línea: 01/04/2018

Para citar este artículo: Limongi, D., Cárdenas-Calle, M. y Erazo, J.C. 2018. Compuestos nitrogenados en los sedimentos del estero San Camilo: Guayas, Ecuador y su relación con actividades antrópicas adyacentes. Intropica 13(1):51-56.

DOI:http://dx.doi.org/ 10.21676/23897864.2338 